\title{
Investigation of Beam Performance Parameters in a Pierce-Type Electron Gun
}

\author{
Ashraf A. El-Saftawy ${ }^{1, *}$, Ahmed Elfalaky ${ }^{2}$, Magdi S. Ragheb ${ }^{1}$, Safwat G. Zakhary ${ }^{1}$ \\ ${ }^{1}$ Accelerators and Ion Sources Department, Nuclear Research Center, Atomic Energy Authority, Cairo, P.O. 13759, Egypt \\ ${ }^{2}$ Physics Department, Faculty of Science,Zagazig University, Zagazig, P.O. 44519, Egypt
}

\begin{abstract}
In the present work, an investigation has been made for the extraction characteristics and beam diagnosis for a Pierce-type electron gun with spherical anode to acquire an electron bea m suitable for different applications. The acceleration voltage increases the electron beam currents up to $250 \mathrm{~mA}$ at Acceleration voltage $75 \mathrm{kV}$ and decreases the beam perveance, beam waist and beam emittance. The minimu mbeam radius could be found at the min imu $\mathrm{m}$ beam perveance and maximum convergence angle. Also the increase of the accelerating voltage affects the increase of the beam fluence rate up to $1.3 \times 10^{18}$ $\mathrm{e} / \mathrm{min} . \mathrm{cm}^{2}$, due to the increase of the extracted current. Tracing the electron beam profile by X-Y probe scanner along the beam line at two different places reveals that the spherical anode affects the beam to be convergent. The electron beam could be suited for the two suggested experiments in our lab, plasma acceleration and surface modifications of poly mers.
\end{abstract}

Keywords Electron Gun, Beam Geo metry, Perveance, Emittance

\section{Introduction}

The development of electron beam devices has a long history and electron beam technology has undergone several changes over the years. A variety of electron beam sources have been designed and utilized in the basic research as well as for the industrial applications[1-3]. The electron beam parameters such as diameter, current, energy, etc. were adjusted to suit their specific experimental requirements [4]. Charged particle beams from different types of sources have to be transmitted without loss of the particles through the accelerator. It is very important that the particles strike the target should have the parameters required for a certain application. For a beam transmission without loss of the particles, the cross-section of the beam must not exceed a given maximum value of a well-defined point. To achieve this we will introduce an important quantity known as emittance which one wants to minimize for any given current[5]. The emittance is an important aspect for high-quality beam, which is basically defined by the product of the width and transverse velocity spread of the beam (region of phase space occupied by beam). If the beam is densely packed, then the emittance is said to low. Whereas beam that is somewhat spread out has high emittance[6-8].

Knowing the beam properties before its injection into the accelerator saves the time needed for accelerator tuning and

* Corresponding author:

aama1978@yahoo.com (Ashraf A. El-Saftawy)

Published online at http://journal.sapub.org/scit

Copyright (C) 2012 Scientific \& Academic Publishing. All Rights Reserved increases the overall efficiency of the beam transmission[9]. At the present work, the electron beam properties will be defined and regulated to match the suggested applications (plasma Acceleration and electron beam treatments of different types of materials). The electron beam diagnostics done in this work include the measurement of electron beam intensity, perveance, profile, and hence emittance as a function the acceleration voltages. The beam fluence and fluence rate will also be considered.

\section{Experimental Setup}

The electron beam is produced from a Pierce-type electron gun (figure 1) with an indirectly heated cathode operating in a diode circuit. The Pierce electrode makes an angle $67.5^{\circ}$ with the beam edge. The cathode, together with the beam shaping electrode, is connected to a pulse modulator which provides negative high voltage (up to $80 \mathrm{kV}$ ) pulses with variable pulse repetition rates (from 31.25 to 500 counts/s) which accelerates the electrons with a velocity of about $0.5 \mathrm{c}$ (the velocity of light). The anode with a spherical shape is grounded to the vacuum chamber i.e. considered positive with respect to the cathode and the beam shaping electrodes. In the field free region behind the anode, a chamber exists having three flanges, figure 1 . The gun section is connected to the field free space chamber through an aperture in the anode electrode. The electron beam scanner, shown in figure 2 , is an electromechanical system with a thin long wire $(0.1 \mathrm{~mm}$ diameter and $100 \mathrm{~mm}$ long) is used for probing the beam to study the dependence of beam parameters on the acceleration voltages. 

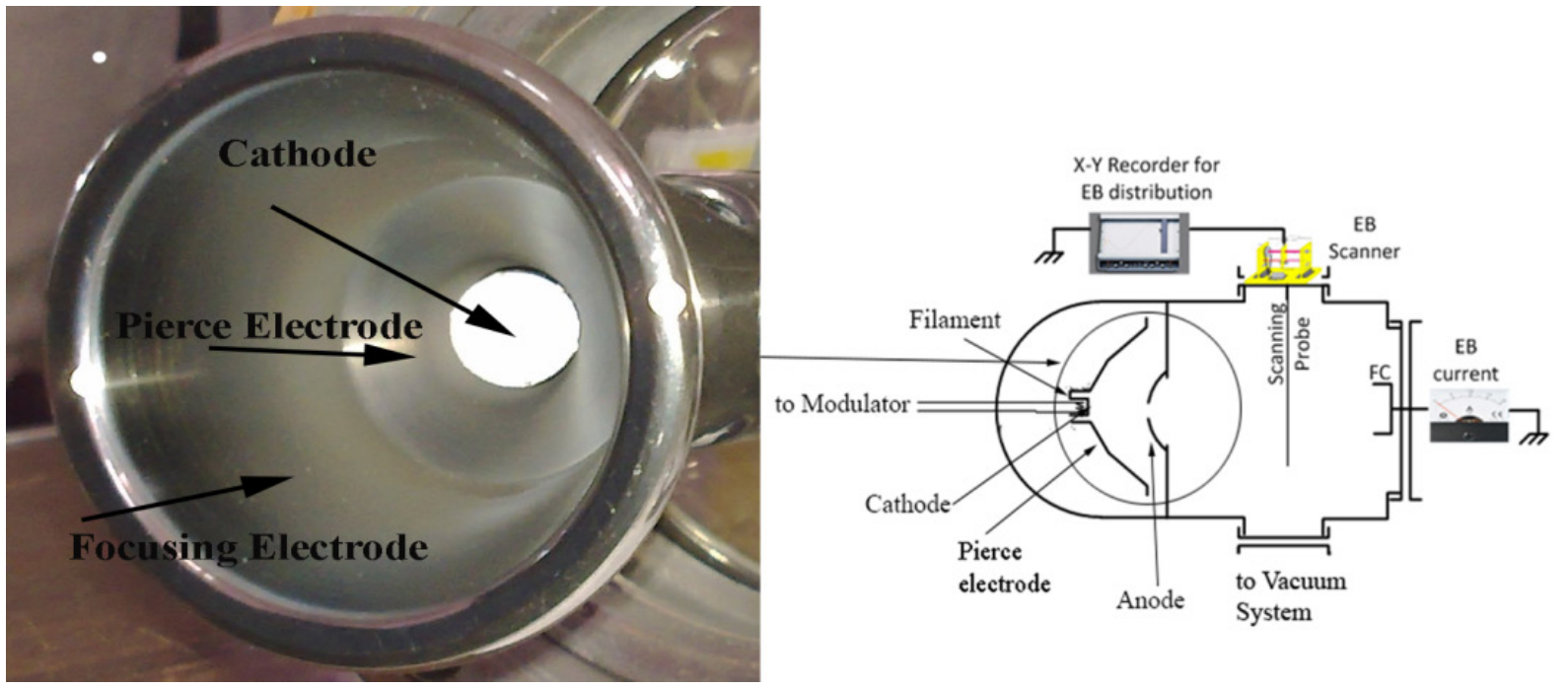

Figure 1. Electron gun configuration

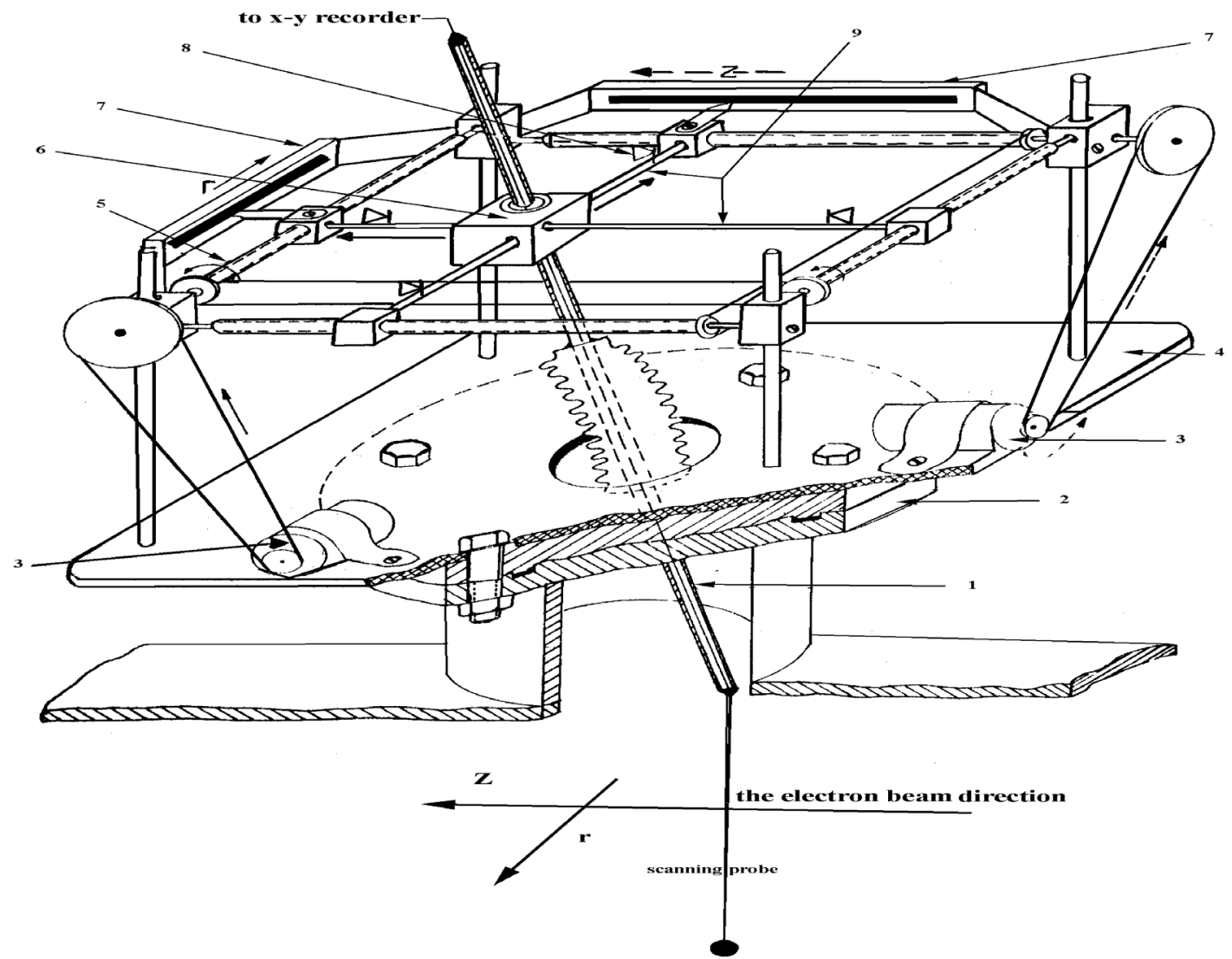

Figure 2. Electron beam scanner (1- Coaxial insulated rod.2-The upper middle flange of the field free region.3-Motors.4- Metallic square sheet. 5- Screw rods.6- Central cuboid.7-Sliding resistance. 8- Micro-switch. 9- Two perpendicular cylindrical rods

The scanning probe moves in the direction of the beam (z-direction) and moves in a direction perpendicular to the beam direction. The scanning probe is placed at distances $20 \mathrm{~mm}$ (at the beam waist) from the anode, and a set of currents $I_{p}$ values is recorded for different Acceleration Voltages. Then the probe is shifted to a new distance $150 \mathrm{~mm}$ (at the beam exit) from the anode, and the previous procedures are repeated. More details about this scanner could be found in literature[10].

\section{Results and Discussion}

\subsection{Studying Electron Beam Intensity and Perveance}

Figure 3 shows the influence of the Acceleration voltages on the extracted electron beam current and perveance. The 
electron beam current increases (from $20 \mathrm{~mA}$ up to $250 \mathrm{~mA}$ ) with the increase of the Acceleration voltage up to $75 \mathrm{kV}$, this dependency is in close agreement with child-Lang muir[11]:

$$
I=7.31 \times 10^{-6}\left(\frac{r_{a}}{d}\right) U^{3 / 2}
$$

Where, $r_{a}$ is the beam radius at the anode exit aperture and $d$ is the distance between the cathode and the anode.

At higher acceleration voltages, the increase of the electron beam current is limited and the current seems to be quasi-saturated in this region. This quasi-saturation effect is due to the fact that, the number of the backscattered electrons increases with increasing the electron energy $[12,13]$. Space charge effect in beams is conveniently characterized by the perveance taking into account the magnitude of the beam current and the acceleration voltage[14].

Figure 1 shows that the perveance decreases with the increase of the acceleration voltage. The beam perveance decreases from $5.7 \times 10^{-2} \mu$ perv down to $1.2 \times 10^{-2} \mu$ perv and the Acceleration voltages increase from $5 \mathrm{kV}$ up to $75 \mathrm{kV}$ respectively. For values of the perveance $\mathrm{P} \leq 10^{-2} \mu$ perv the intrinsic electric field of the beam has an appreciable effect on the motions of the electrons. But for values of $\mathrm{P} \geq$ $10^{4}$ uperv, the effect of space charge can be neglected[15].

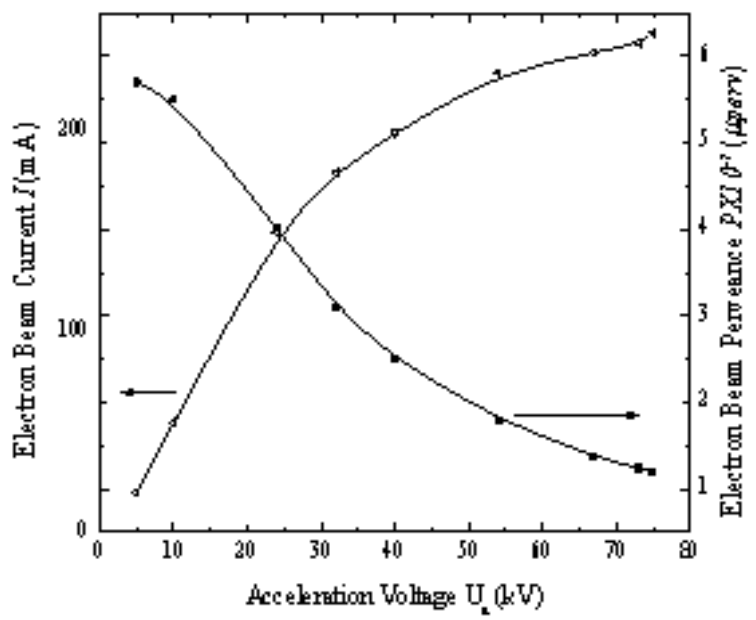

Figure 3. Electron beam current and perveance as a function of acceleration voltage

\subsection{Electron Beam Profile and Emi ttance Analysis}

Here we will trace the beam profile which describes the current intensity distribution, the beam width, and the angular divergence of the beam. For plas ma acceleration experiment, the injected electron beam has to be very thin, with high convergence angle and very low emittance. These conditions are achieved at the electron beam waist which is located at a distance $20 \mathrm{~mm}$ fro $\mathrm{m}$ the anode aperture. So that, the electron beam profile is traced at this point to find the optimum conditions for performing the plasma acceleration experiments. The traced profile (at acceleration voltages $36 \mathrm{kV}, 48 \mathrm{kV}, 60 \mathrm{kV}$ and $75 \mathrm{kV}$ ) is shown in figure $4 \mathrm{a}$ which is fitted to the Gaussian function, and the fitting parameters are shown in table 1 .
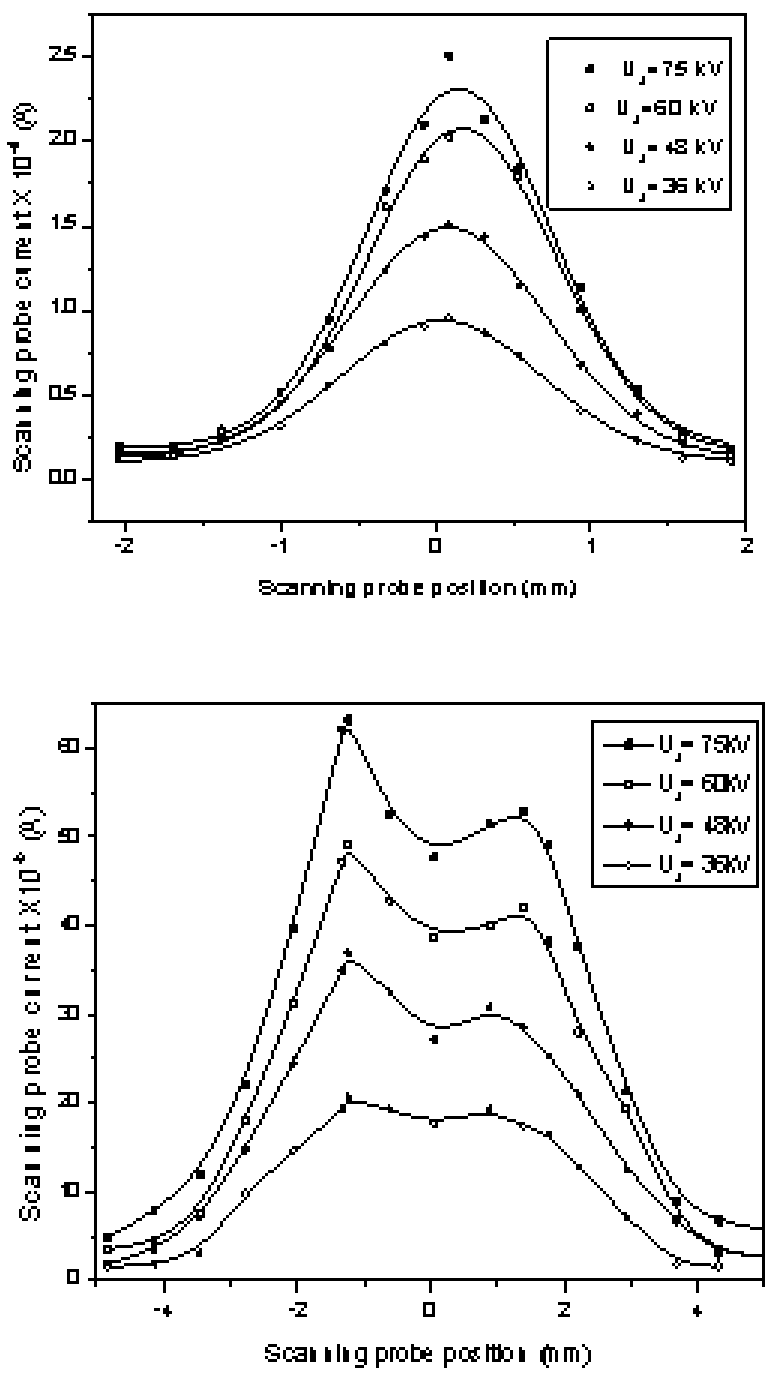

Figure 4. Electron beam profile traced at a) $20 \mathrm{~mm}$ and b) $150 \mathrm{~mm}$ from the anode aperture

The produced beam has to be wide to match the experiment of electron beam treatments of materials for surface modification. The electron beam is found to be wider and the convergence angle is larger and hence the beam emittance is larger at the Faraday Cup (FC) i.e. target holder. So that the beam profile is traced at distance $150 \mathrm{~mm}$ fro $\mathrm{m}$ the anode aperture as shown in figure $4 \mathrm{~b}$. This figure shows two peaks which are fitted Gaussian and shown in figure 5. The deduced fitting data are also summarized in table 1.

Table 1 shows the variations of the beam parameters with different beam energies. In such a table we can see the parameters; $x_{c}$ is the centre of the beam, $w$ is the beam full width at half maximu (FWHM) and $A$ is the area under the curve. Table 1 shows that;1) the beam width decreases with energy i.e. raising beam energy focuses the beam.2) Electron beam current intensity increases with the beam energy; this is proved by the increasing of $A$ and the increase $y_{c}$ (the beam height).3) The beam centre changes and deviates from zero point; this may be due to the strong repulsive force which increases as the electrons become closer to each other i.e. the beam is focused [16]. 

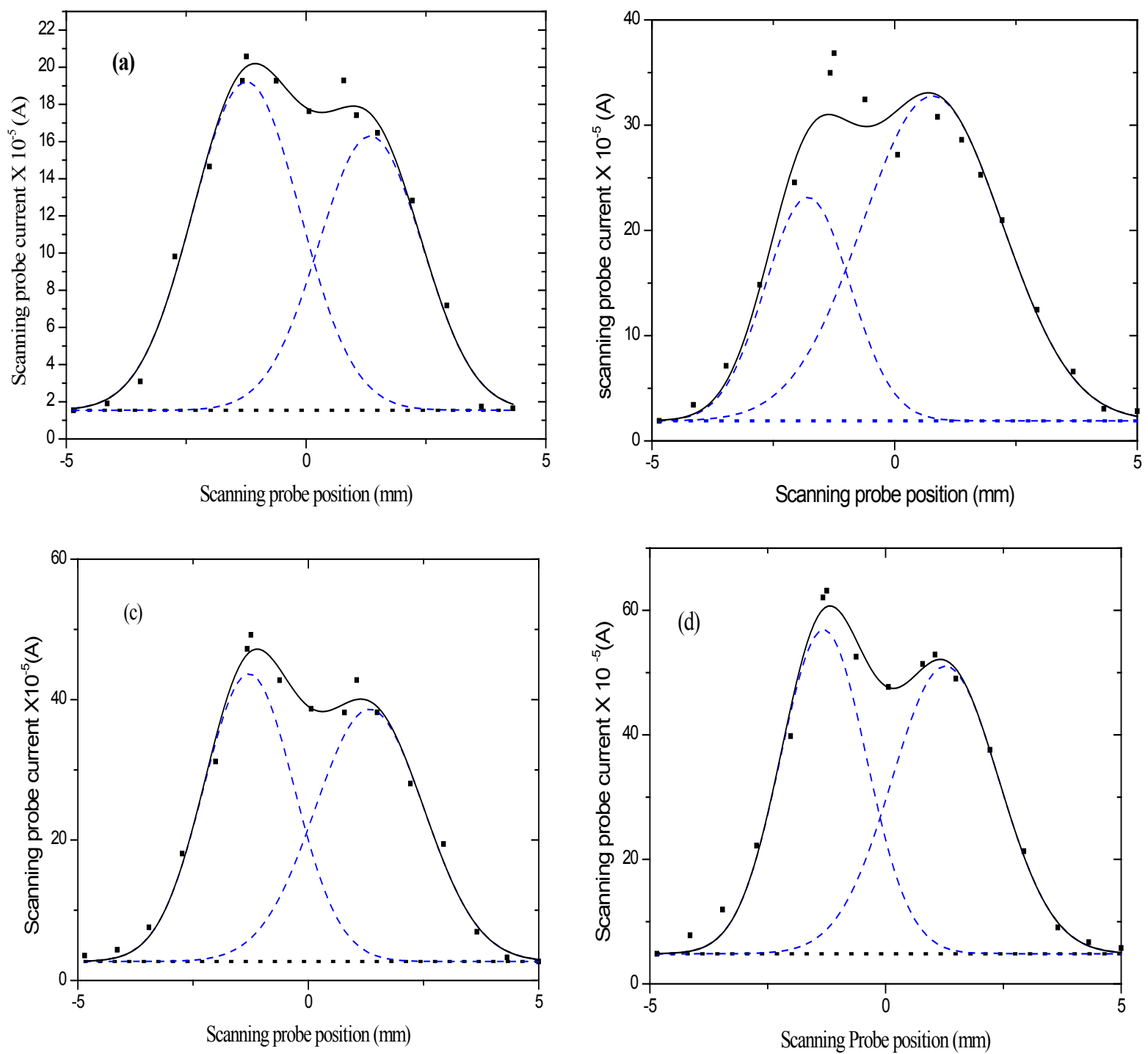

Figure 5. Gaussian fit for beam profile traced at $150 \mathrm{~mm}$ for different acceleration voltages; a) $36 \mathrm{kV}$, b) $48 \mathrm{kV}$, c) $60 \mathrm{kV}$ and d) $75 \mathrm{kV}$., Where( )is the cumulative fit peak, (----) is the fit peak 1 and 2 , and (.....) is the base line

Table 1. Beam fitting parameters

\begin{tabular}{|c|c|c|c|c|c|c|c|}
\hline Energy $(\mathrm{keV})$ & $x_{c}$ & $A$ & $w$ & & $x_{c}$ & $A$ & $w$ \\
\hline & \multicolumn{3}{|c|}{ Data extracted from figure $4 a$. } & \multicolumn{4}{|c|}{ Data extracted from figure 5.} \\
\hline 36 & 0.04 & 1.36 & 1.3 & $\begin{array}{l}\text { Peak1 } \\
\text { Peak2 }\end{array}$ & $\begin{array}{l}-1.27 \\
1.33\end{array}$ & $\begin{array}{l}49.3 \\
41.6\end{array}$ & $\begin{array}{l}2.64 \\
2.50\end{array}$ \\
\hline 48 & 0.08 & 2.2 & 1.28 & $\begin{array}{l}\text { Peak1 } \\
\text { Peak2 }\end{array}$ & $\begin{array}{c}-1.79 \\
0.78\end{array}$ & $\begin{array}{c}46.5 \\
111.6\end{array}$ & $\begin{array}{l}2.06 \\
3.40\end{array}$ \\
\hline 60 & 0.17 & 2.9 & 1.21 & $\begin{array}{l}\text { Peak1 } \\
\text { Peak2 }\end{array}$ & $\begin{array}{c}-1.28 \\
1.37\end{array}$ & $\begin{array}{l}105.9 \\
104.9\end{array}$ & $\begin{array}{l}2.36 \\
2.67\end{array}$ \\
\hline 75 & 0.15 & 3.16 & 1.19 & $\begin{array}{l}\text { Peak1 } \\
\text { Peak2 }\end{array}$ & $\begin{array}{c}-1.28 \\
1.36\end{array}$ & $\begin{array}{l}129.6 \\
124.4\end{array}$ & $\begin{array}{l}2.27 \\
2.50\end{array}$ \\
\hline
\end{tabular}

Increasing the acceleration voltages, increases the axial component of the accelerating field with respect to the transverse component which affects the beam to spread and thus allowing the beam to become more convergent[17]. The velocities of the electrons emitted perpendicular to the cathode surface have two components; axial and transverse.
The axial component $v_{z}$ is proportional to the accelerating energy $e U$ where [18];

$$
v_{z}=\sqrt{\frac{2 e U}{m}}
$$

The transverse component $v_{x}$ is proportional to the 
transverse kinetic energy $k T(k$ is Boltzmann constant and $T$ is the electrons temperature) of the electrons which causes the spread of the beam width, where[19]:

$$
v_{x}=\sqrt{\frac{k T}{m}}
$$

Therefore, the angle of beam divergence $\gamma$ is found to be in the form[20];

$$
\gamma=\sqrt{\frac{k T}{e U}}
$$

Since $e U$ is the axial accelerating energy of the electron beam. From such an equation, it is seen that $\gamma$ is proportional to the electron temperature and invers ely proportional to the accelerating voltage $U$, i.e. the increase of the anode voltage causes a decrease of the beam width and emittance. As the electrons approach the axis of the beam, the columbic repulsive force increases, this effect leads to the escape of electrons towards the beam boundaries. This phenomenon explains the splitting of the beam profile curves shown in figure $4 \mathrm{~b}$. Under the action of the anode lens these two peaks disappear, figure 4a. After the beam escapes from the anode lens field, the beam splitting appears, figure $4 \mathrm{~b}$.

Figure 6 shows the variation of the beam radius at waist and at the FC entrance with the variation of the electron beam perveance. The electron beam radius increases with the increase of the electron beam perveance. Hence to obtain a minimu meam waist we have to use a low perveance beam. These results are in a very close agreement with the computer simulation results previously reported[21]. Finally, the increase of the beam width with the increase of $z$ and the decrease of this width with the increasing of the extracting voltages are evident from figures 4 and 5 . The space charge effect is believed to be the cause of the divergence of the electron beam; while the focusing property of the anode aperture imp roves the beam profile confinement i.e. focuses the electron beam.

The emittance is being roughly proportional to the ratio of trans verse velocity to a xial velocity of the electron. This rat io gives the slope of the trajectory leaving the emitting surface. If the emitting surface is a circle with radius $r_{c}$, then the normalized emittance is defined as[19]:

$$
\varepsilon_{n}=2 r_{c}\left(\frac{k T}{m}\right)
$$

And the trans verse emittance $\varepsilon_{x}$ is given by:

$$
\varepsilon_{x}=A(X, \dot{X}) / \pi
$$

The envelope of $A(X, \dot{X})$ is an upright ellipse with extreme values $\left(x_{m}, x_{m}\right)$; then its area is $\left(\pi x_{m} x_{m}\right)$, so:

$$
\varepsilon_{x}=x_{m} x_{m}=r \gamma
$$

For small values of $\tan \gamma=\gamma=r / z$, Where; $r$ is the beam radius and $z$ is the distance from the exit aperture of the beam to the scanning probe. The increase of the acceleration voltage decreases the width and the divergence angle of the electron beam and hence the electron beam emittance decreases. Table (2) confirms the principle which states that theminimum beam radius is found at minimum beam perveance and higher beam convergence.

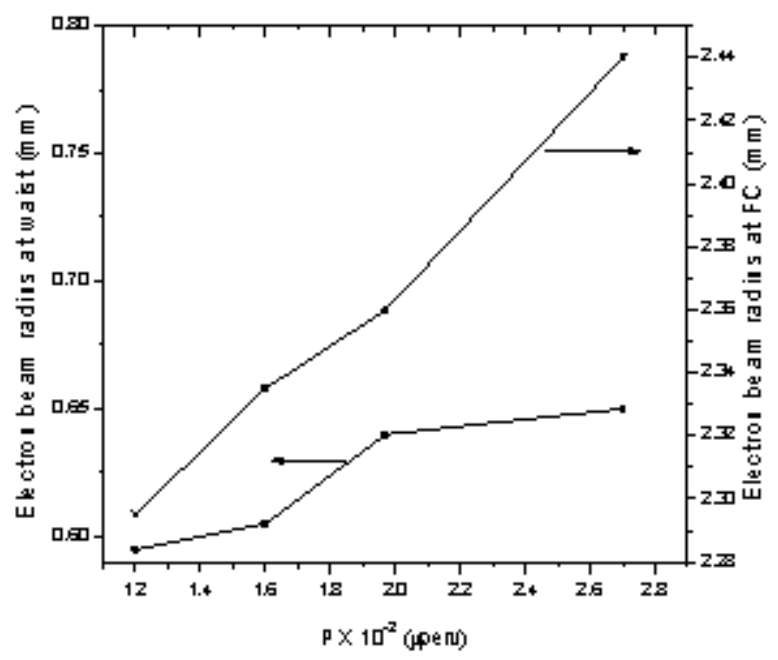

Figure 6. Beam radius at waist and Faraday cup variation with beam perveance

Table 2. Variation of beam geometry and emittance with energy.

\begin{tabular}{|c|c|c|c|}
\hline $\begin{array}{c}\text { Energy } \\
\mathrm{keV}\end{array}$ & $\begin{array}{c}r_{1} \\
\mathrm{~mm}\end{array}$ & $\begin{array}{c}\gamma_{1} \\
\mathrm{mrad}\end{array}$ & $\begin{array}{c}\varepsilon_{1} \\
\mathrm{~mm} . \mathrm{mrad}\end{array}$ \\
\hline 36 & 0.65 & 0.012 & 0.0078 \\
\hline 48 & 0.64 & 0.0119 & 0.0076 \\
\hline 60 & 0.605 & 0.0112 & 0.0068 \\
\hline 75 & 0.595 & 0.011 & 0.0065 \\
\hline & $r_{2}$ & $\gamma_{2}$ & $\begin{array}{c}\varepsilon_{2} \\
\mathrm{~mm}\end{array}$ \\
$\mathrm{mrad}$ & $\mathrm{mm} . \mathrm{mrad}$ \\
\hline 36 & 2.44 & 0.0163 & 0.04 \\
\hline 48 & 2.36 & 0.0157 & 0.037 \\
\hline 60 & 2.335 & 0.0155 & 0.036 \\
\hline 75 & 2.295 & 0.015 & 0.034 \\
\hline
\end{tabular}

In table (2), $\left(r_{1}, \gamma_{1}, \varepsilon_{1}\right)$ are the beam radius, convergence angle and emittance at waist, and $\left(r_{2}, \gamma_{2}, \varepsilon_{2}\right)$ are the beam radius, convergence angle and emittance at the entrance of FC.

\subsection{Electron Beam Fluence Calculation}

Electron beam absorbed dose is used to identify the electron beam radiation in the science of dosimetry. Also the following quantities are used to describe an ionizing radiation beam (electron beam): particle fluence, energy fluence, particle fluence rate and energy fluence rate. These quantities are usually used to describe charged particle beam[22, 23]. In this work we will use the terms, electron beam fluence and fluence rate to describe the electron beam in the irradiation of materials surfaces experiments. According to the definitions of fluence and fluence rate, 
these parameters are calculated and shown in figures 7 and 8 .

The particle fluence $\phi$ (measured in $\mathrm{m}^{-2}$ ) is defined as the number of particles $d N$ incident on cross-sectional area $d A$ and could be expressed as in the formula; $\phi=d N / d A$, While the particle fluence rate $\phi^{\prime}$ (measured in $\mathrm{m}^{-2} \mathrm{~s}^{-1}$ ) is the increment of the fluence $d \phi$ in time interval $d t$ and could be expressed as $\phi^{\prime}=d \phi d t$.

Figure 7 shows the increase of the fluence rate (number of electrons incident on a specific area per second) with the increase of the acceleration voltage. The maximum number of electrons enter the FC are $1.56 \times 10^{18}$ electrons at acceleration voltage $75 \mathrm{kV}$. The fluence rate is in the range from $\left(2 \times 10^{16}\right.$ up to $\left.2.21 \times 10^{17}\right)$ electrons $/ \mathrm{s} . \mathrm{cm}^{2}$ at energies between $5 \mathrm{keV}$ and $75 \mathrm{keV}$ respectively.According to the irradiation time the electron beam fluence is calibrated at electron beam energy $75 \mathrm{keV}$. Figure 8 shows a straight line, its slope $\left(1.3 \times 10^{18} \mathrm{e} / \mathrm{min} . \mathrm{cm}^{2}\right)$ represents the electron beam fluence rate.

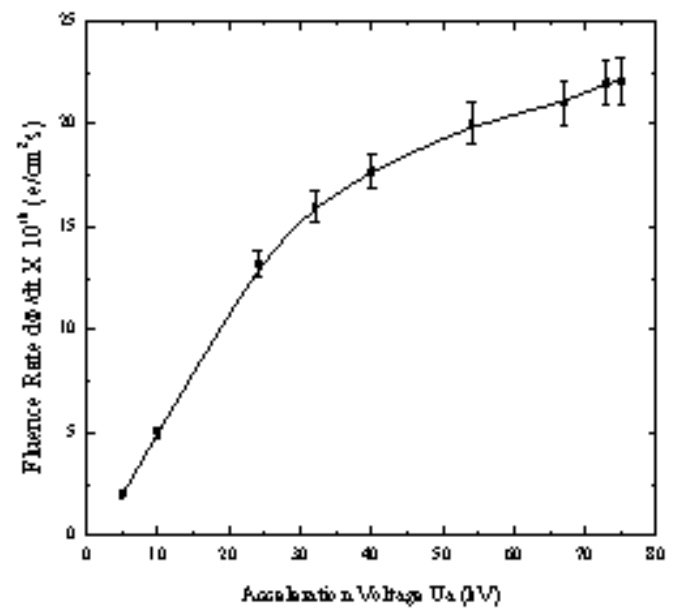

Figure 7. Fluence rate as a function of acceleration voltage

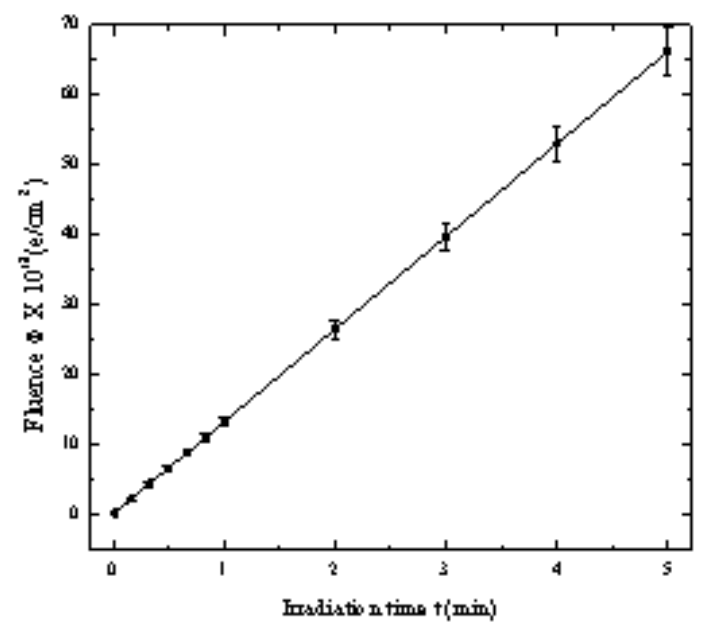

Figure 8. Calibration of electron beam fluence at $75 \mathrm{keV}$

\section{Conclusions}

Different types of electron guns have been designed for producing different electron beam energies. In this work, a Pierce-type electron gun with spherical anode has been investigated to produce an electron beam with suitable properties valid for the suggested application in our laboratory.

Upon investigation of the designed gun, it has been found that the acceleration voltage increases the electron beam currents up to $250 \mathrm{~mA}$ and decreases the beam perveance. The spherical extraction electrode (anode) is believed to produce a convergent beam. This effect is proved by tracing the electron beam density distribution at two different places along the beam line. Also it is found that the distribution of the electron bea $m$ affected by the acceleration voltage i.e. the electron beam diameter increases with the decrease of the acceleration voltage. So that the higher the acceleration voltage, the lower the beam emittance.

Electron beam emittance is preferably to be as low as possible for the suggested plasma acceleration experiment. While the polymer treatment experiment requires a spread out beam with larger diameter i.e. with high emittance. It is proven that the min imum beam radius could be found at the minimum beam perveance and maximum convergence angle.

Also the electron beam fluence rate and electron fluence are considered and calculated, these two values depend on the acceleration voltage. We can conclude that the resulting electron beam (density and shape) could be controlled by not only the gun geometry but also the acceleration voltages to produce a suitable electron beam for different applications.

\section{REFERENCES}

[1] R. Bakish, "Electron Beam Technology”, Wiley, New York, 1962.

[2] A.V. Deore, B.J. Patil, V.N. Bhoraskar, S.D. Dhole, “Design, Development and Characterization of Tetrode Type Electron Gun System for Generation of Low energy Electrons", Indian Journal of Pure \& Applied Physics, Vol. 50, 482-485, 2012.

[3] R. Mehnert, "Review of Industrial Applications of Electron Accelerators", Nucl. Instr. and Meth. B 113, 81-87, 1996.

[4] S.K. Mahapatra, S.D. Dhole and V.N. Bhoraskar, "A $20 \mathrm{keV}$ Electron Gun System for the Electron Irradiation Experiment", Nucl. Instr. and Meth in Phys. Res. A 536, 222-225, 2005

[5] S. Humphries Jr., "Charged Particle Beams", Wiley, New York, 1990

[6] M. Reiser, "Theory \& Design of Charged Particle Beams", Wiley”, New York, 1994.

[7] G. Herrmann, "Optical Theory of Thermal Velocity Effects in Cylindrical Electron Beams", J. Appl. Phys. 29 (2), 127, 1958.

[8] K. Masood, M. Iqbal and M. Zakaullah, "Emission Characteristics of the Thermionic Electron Beam Sources 
Developed at EBSDL", Nucl. Instr. and Meth in Phys. Res. A 584, 9, 2008.

[9] M.S. Ragheb and M.H.S. Bakr, "Improvement of Auger Spectra Using A.C. Modulation Technique", Radiat. Phys. Chem. 47 (5), 673-675, 1996.

[10] M.H.S. Bakr and M.S. Ragheb, "the Electron Injector IV. A Remote Tracer for the Electron Beam Current", Arab Republic of Egypt, Atomic Energy Establishment (AREAEE) / Rep. 262, 1-16, 1981.

[11] S.I. Molokovsky and A.D. Sushkov, "Intense Electron and Ion Beams", Springer-Verlag, Berlin, 2005.

[12] A.M.D. Assa'd and M.M. El-Gomati, "Backscattered Coefficients for Low Energy Electrons", Scanning Microscopy Vol. 12, No. 1, 185 - 192, 1998.

[13] R.K. Yadav and R. Shanker, "Contribution of Backscattered Electrons to the Total Electron Yield Produced in Collisions of 8-28 keV Electrons with Tungsten", PRAMANA J. of Physics, Vol. 68, No 3, 507-515, 2007.

[14] A.A. El-Saftawy, A.A. Hassan, M.M. Abdelbaki, S.G. Zakhary and Z. Awaad, "Enhancement of Axial Type RF Source Characteristics", Arab J. Nucl. Sci. Appl., 39 (2), 189-197, 2006.

[15] A. Zhigarev; "Electron Optics and Electron Beam Devices", Mir Publisher, Moscow, 1975.
[16] P. Tippler, "Physics for Scientists and Engineers: Electricity, Magnetism, Light, and Elementary Modern Physics", (5th ed.). W. H. Freeman, 2004.

[17] M.E. Abdelaziz, S.G. Zakhary and M.S. Ragheb, "Beam diagnostics extracted from radio frequency driven ion source", Rev. Sci. Instrum. 71,2, 1137-1139, 2000.

[18] S.G. Zakhary, "Beam Diagnostic Technique for a Small-Size High-Efficiency Radio-Frequency Ion Source", Rev. Sci. Instrum. 66, 12, 1995.

[19] A.A. El-Saftawy, "Factors Enhancing the Ion Beam Optics and Transport in the Low Energy Accelerator (50keV)", M.Sc. Thesis, Faculty of Science, Zagazig University, 2007.

[20] T.S. Green, "Beam Formation and Space Charge Neutralization”, IEEE Trans. Nucl. Sci. 23 (2), 918-928, 1976.

[21] A. Hassan, A. Elsaftawy and S.G. Zakhary, "Analytical Studies of the Plasma Extraction Electrodes and Ion Beam Formation", Nucl. Instr. and Meth. in Phys. Res. A 586, $148-152,2008$

[22] B. Nilsson, A. Montelius and P. Andreo, "Determination of fluence correction factors in electron beam dosimetry", Radiother. Oncol. 37, 1995.

[23] D.T. Burns, G.X. Ding and D.W.O. Rogers," R50 as a Beam Quality Specifier for Selecting Stopping Power Ratios and Reference Depths for Electron Dosimetry", Med. Phys. 2, 383-388, 1996. 